\title{
Quel avenir pour l'éolien communautaire et les autres énergies renouvelables au Québec : pourquoi pas des tarifs d'achat garanti?
}

\author{
Évariste Feurtey, Doctorant en sciences de l'environnement \\ Université du Québec à Rimouski
}

\section{INTRODUCTION}

La stratégie énergétique 2006-2015 du gouvernement québécois prévoit implanter 4000 MW d'énergie éolienne sur le réseau d'Hydro-Québec (HQ) d'ici 2015.

La stratégie énergétique 2006-2015 du gouvernement québécois prévoit implanter $4000 \mathrm{MW}$ d'énergie éolienne sur le réseau d'HydroQuébec (HQ) d'ici 2015². En 2012, à l'heure d'un premier bilan, il s'avère que certains projets retenus ne remplissent pas toutes leurs promesses tant en terme d'acceptabilité sociale ou de retombées. C'est ainsi que près de $700 \mathrm{MW}$ de projets éoliens ne verront finalement pas le jour. De plus, les collectivités locales attendent que le gouvernement annonce officiellement un programme de microproduction pour les différentes énergies renouvelables (éolien, photovoltaïque, bioénergie, etc.) comme cela était prévu dans le cadre de la stratégie énergétique de 2006-2015. En attendant, il ne leur est pas possible de se brancher au réseau d'HydroQuébec pour vendre l'électricité autrement qu'en passant par un processus d'appel d'offres compétitif très coûteux. Dans ces conditions, par quels mécanismes tarifaires passe l'avenir de l'éolien et des autres énergies renouvelables au Québec, notamment en région habitée?

Le gouvernement a répondu en partie à ces interrogations en juillet 2011 en annonçant la réalisation de deux appels d'offres supplémentaires : le premier de $700 \mathrm{MW}$ en région habitée pour combler les MW non réalisés dans le cadre de la politique énergétique actuelle, et le second de $300 \mathrm{MW}$ dans le Nord-du-Québec en complémentarité du Plan Nord. Ces nouveaux appels d'offres seraient lancés en 2012. Enfin, on attend encore la diffusion du rapport de l'équipe spéciale de Steven Guilbault, demandée à l'époque par la ministre des Ressources naturelles, madame Normandeau, pour statuer sur le sort réservé aux énergies émergentes et à la microproduction attendue initialement pour l'automne 2010. Comme le dévoile Louis-Gilles Francoeur dans un article du 31 décembre 2011, celui-ci est sur la table du ministère depuis décembre 2010 et préconiserait des «tarifs d'achat garanti » plafonnés en volume pour toutes les énergies émergentes au Québec ${ }^{2}$. Pourquoi n'est-il pas encore diffusé par le gouvernement? Cette recommandation gêne-telle les décideurs politiques?

Devant cette logique «implacable » des appels d'offres et le climat décisionnel incertain concernant la microproduction, de nombreuses personnes ou organismes remettent en question la seule perspective des appels d'offres pour l'avenir énergétique du Québec en région habitée. N'y aurait-il pas place pour une tarification mixte au Québec? Ne serait-il pas possible d'envisager de gros projets par appel d'offres dans le Nord québécois, en zone peu habitée et de développer des petits projets d'origine renouvelable communautaires ou de microproduction avec des tarifs d'achat garanti proche des lieux de consommation d'énergie? C'est en tout cas le souhait de plusieurs auteurs ${ }^{3}$ dans une lettre ouverte parue dans le Devoir en date du 10 février 2012.

Cet article vise à expliquer et démystifier pourquoi les tarifs d'achat garanti se développent de par le monde pour le développement des énergies renouvelables et quels sont leurs avantages ou inconvénients par rapport aux appels d'offres. L'idée est de faire réaliser aux Québécois l'intérêt qu'ils peuvent présenter par rapport au seul mécanisme tarifaire utilisé jusqu'à présent : les appels d'offres. 


\section{PRÉSENTATION DES DEUX FAMILLES TARIFAIRES}

\section{Dans le premier système des tarifs d'achat garanti, les politiciens fixent le prix et la quantité d'énergie produite est déterminée par le marché. Le prix d'achat obtenu est le même pour tous les investisseurs potentiels. Il est connu et garanti pour toute la durée du contrat d'achat d'électricité (en général de 15 à 25 ans).}

Les systèmes tarifaires déterminent les prix de vente de l'énergie éolienne et les conditions d'accès au réseau électrique, ce qui constitue les deux composantes essentielles du développement technologique ${ }^{4}$. À l'heure actuelle, il existe deux familles pour compléter les objectifs de croissance de la capacité de production. D'abord, les tarifs d'achat garanti, communément appelé feed in tariff (FIT) en anglais, ont été inventés par les pays pionniers du développement éolien (Allemagne, Danemark à ses débuts, puis Espagne). Ils sont aujourd'hui utilisés en Allemagne, en Espagne, en France (projets inférieurs à $50 \mathrm{MW}$ ), en Chine, au Portugal, au Royaume-Uni (projets inférieurs à $5 \mathrm{MW}$ ), en Ontario, au Nouveau-Brunswick (projets inférieurs à $200 \mathrm{~kW}$ ) et dans 12 États américains. Ensuite, il y a les politiques par quotas (QUOTAS) ou appels d'offres. Ceux-ci sont principalement utilisés dans les pays anglo-saxons comme le Royaume-Uni (projets supérieurs à $5 \mathrm{MW}$ ), l'Italie, le Québec, d'autres provinces canadiennes et de nombreux États américains.

Dans le premier système des tarifs d'achat garanti, les politiciens fixent le prix et la quantité d'énergie produite est déterminée par le marché $^{5}$. Le prix d'achat obtenu est le même pour tous les investisseurs potentiels. Il est connu et garanti pour toute la durée du contrat d'achat d'électricité (en général de 15 à 25 ans). Il existe maintenant une version améliorée de ce type de tarification (les tarifs avancés en énergie renouvelable). Au terme d'une certaine durée d'exploitation, le prix d'achat de l'énergie décroît plus ou moins en fonction de la qualité du gisement éolien, qui permet de mieux répartir les parcs éoliens sur le territoire.

Dans le deuxième système tarifaire, les QUOTAS, les quantités à produire sont fixées politiquement, et les prix d'achat sont déterminés par le marché. forme les quotas ou l'objectif à atteindre dans un intervalle de temps négocié (par exemple au Québec, on vise à atteindre un quota d'éolien de 4000 MW d'ici 2015). Ce type de politique peutêtre combiné à la délivrance de certification verte. Dans ce cas-là, une partie du prix de vente de l'énergie est fixée par le marché boursier du carbone. Dans les QUOTAS, les contrats d'achat peuvent être attribués par enchère au meilleur coût (appel d'offres). Ainsi au Québec, avec la réglementation actuelle, il n'est pas possible pour un agriculteur ou une municipalité de se brancher au réseau d'Hydro-Québec sans passer par un appel d'offres compétitif.

Dans le deuxième système tarifaire, les

QUOTAS, les quantités à produire sont fixées politiquement, et les prix d'achat sont déterminés par le marché. La quantité demandée forme les quotas ou l'objectif à atteindre dans un intervalle de temps négocié (par exemple au Québec, on vise à atteindre un quota d'éolien de 4000 MW d'ici 2015).

\section{COMPARAISON DES MÉCANISMES TARIFAIRES}

Cette analyse des avantages et inconvénients des deux familles tarifaires se base principalement sur une étude ${ }^{6}$, qui fait la comparaison des deux mécanismes de soutien tarifaire utilisés dans plusieurs pays européens en fonction de huit critères d'évaluation. Nous les présentons ici en y ajoutant quelques données tirées des expériences québécoise et ontarienne, ce qui nous a amenés à remplacer le troisième critère par un critère plus général portant sur «l'acceptabilité sociale» des projets éoliens. Sur ce point, le tableau 1 présente une synthèse tirée de la littérature récente dans ce domaine ${ }^{7}$. 
Tableau 1 - Comparaison des avantages et inconvénients des deux familles de mécanismes tarifaires

\begin{tabular}{|c|c|c|}
\hline \multirow{2}{*}{ Critères d'analyse } & FIT & QUOTAS \\
\hline & Caractéristiques & Caractéristiques \\
\hline 1 - Capacité installée & - Meilleur passé historique & - Peu de systèmes efficaces \\
\hline \multirow{4}{*}{2 - Innovation, industrie et bénéfices } & - Favorise l'innovation (profits élevés $\rightarrow$ RD\&D) & \multirow{4}{*}{$\begin{array}{l}\text { - Favorise l'instabilité des marchés (évolution en dent de scie) } \\
\text { - Perspective à court terme (emplois et bénéfices limités) }\end{array}$} \\
\hline & - Meilleur passé historique pour : & \\
\hline & - la création d'un marché local et d'emplois & \\
\hline & - la création d'une industrie manufacturière nationale & \\
\hline \multirow[t]{8}{*}{3 - Acceptabilité sociale } & - Initiatives «bottom up» encouragées et favorisées & - Initiatives «exogènes» favorisées \\
\hline & - Information complète et transparente sur les projets & - Information incomplète avant l'acceptation du projet \\
\hline & - Projets soumis quand ils sont prêts & - Temps imparti \\
\hline & - Distribution géographique possible & - Concentration des projets sur les meilleurs sites \\
\hline & - Équité du mode de propriété & - Favorise les gros promoteurs au détriment des petits \\
\hline & - Taille de projets diversifiée & - Favorise les projets de grandes tailles \\
\hline & - Marge de manoeuvre élevée $\rightarrow$ retombés locales importantes & - Moindre coût $\rightarrow$ retombés locales faibles \\
\hline & $\rightarrow$ Favorise l'acceptabilité locale & $\rightarrow$ Acceptabilité locale difficile \\
\hline \multirow[t]{2}{*}{4 - Diversité technologique } & - Différentiation technologique possible & \\
\hline & - Adéquation avec les premières phases du marché & - Favorise les technologies étrangères et existantes \\
\hline \multirow{3}{*}{5 - Coûts, prix et concurrence } & & - Pas forcément les coûts les plus faibles \\
\hline & - Compétition manufacturière $\rightarrow$ diversité d'acteurs & - Compétition $\rightarrow$ peu de joueurs sur le marché \\
\hline & - Réduction des coûts avec les années & - Réduction ou augmentation des coûts avec les années \\
\hline \multirow{2}{*}{6 - Sécurité financière } & - Certitude des tarifs connus d'avance sur 20 ans & - Niveaux d'incertitudes et de risques élevés \\
\hline & - Financement facile auprès des banques & - Coûts élevés de soumission aux appels d'offres \\
\hline 7 - Facilité d'implantation & $\begin{array}{l}\text { - Simple à administrer et à respecter (seul le prix est à fixer) } \\
\text { - Transparence }\end{array}$ & $\begin{array}{l}\text { - Complexe à mettre en oeuvre } \\
\text { - Fraudes possibles }\end{array}$ \\
\hline 8 - Flexibilité & $\begin{array}{l}\text { - Évolutifs (révisions et ajustements possibles) } \\
\text { - Pas de limite maximale fixée (dépasement possible) }\end{array}$ & $\begin{array}{l}\text { - Quotas et échéanciers difficilement ajustables } \\
\text { - Demande prévisible }\end{array}$ \\
\hline
\end{tabular}

\section{Critère 1 et 7 : Capacité installée et facilité d'administration}

Les tarifs d'achat garanti sont simples à implanter et présentent le meilleur passé historique pour diffuser rapidement les technologies renouvelables de par le monde : ils remplissent presque systématiquement leurs promesses en terme de capacité installée.

Les tarifs d'achat garanti sont simples à implanter et présentent le meilleur passé historique pour diffuser rapidement les technologies renouvelables de par le monde : ils remplissent presque systématiquement leurs promesses en terme de capacité installée. À contrario, les QUOTAS sont complexes et longs à mettre en œuvre; ils ont souvent des difficultés à remplir leurs promesses en termes de capacité installée.

En Europe, l'Espagne, le Danemark et l'Allemagne réalisaient en $200380 \%$ des approvisionnent en énergie éolienne ${ }^{6}$; tous ces pays ont amorcé leur développement éolien par des tarifs d'achat garanti. Plus récemment, le cas du Portugal est lui aussi très intéressant. Ce pays est en train de devenir l'un des leaders européens dans les énergies renouvelables grâce à la combinaison d'une politique tarifaire agressive basée sur des tarifs d'achat garanti et de subventions directes à l'établissement de projets. Implanté véritablement en 2005, cette politique incitative a permis au Portugal de produire près de $36 \%$ de son électricité à partir d'éolien et de photovoltaïque en 2010 (ce taux était de $17 \%$ en 2001).

Pour des politiques par quotas, les résultats sont beaucoup plus mitigés et il est rare que les pays réussissent leurs promesses en termes de mégawatts installés. C'est le cas du RoyaumeUni depuis les années 90. Le Québec ne fait pas exception à cette règle. Comme nous l'avons déjà mentionné, des 4000 MW acceptés dans le cadre des trois premiers appels d'offres, près de $700 \mathrm{MW}$ de projets ne se construiront pas. C'est ce qui explique pourquoi le gouvernement doit relancer de nouveaux appels d'offres pour réaliser son objectif de $4000 \mathrm{MW}$. Au contraire, depuis que les tarifs d'achat ont été instaurés et différentiés en Ontario pour les filières énergétiques renouvelables en 2006 et bonifiés en 2009, le programme remplit pleinement ses espérances: la capacité éolienne installée a rapidement progressé en quelques années pour devenir deux fois plus importante qu'au Québec en 2010. 
Une des raisons de l'efficacité importante des tarifs d'achat garanti tient certainement à leur facilité d'administration et à leur transparence; ils ne requièrent que la fixation de tarifs adéquats pour être implantés. Une fois que le système est établi, le gouvernement a seulement besoin de réaliser un ajustement régulier des tarifs. Au contraire, les politiques par quotas sont beaucoup plus difficiles, complexes et coûteuses à mettre en œuvre. Les décideurs politiques doivent établir des cibles et échéanciers de réalisation, déterminer quelles technologies (type et échelle) sont qualifiées pour cela et assurer la gestion du système. Par exemple, les agences gouvernementales doivent définir les pénalités en cas de non-respect des contrats et collecter les pénalités. Elles doivent aussi certifier les producteurs d'énergies renouvelables, déterminer quelles catégories d'acteurs seront impliquées et définir l'échéancier et les objectifs de réalisation. Afin de réussir ce dernier choix, il leur est important de comprendre les coûts et courbes d'apprentissage pour les énergies renouvelables pertinentes. Toutes ces décisions déterminent l'impact des QUOTAS et rendent difficile l'implantation d'un tel système ${ }^{6}$.

Ainsi, les appels d'offres sont généralement longs à mener à terme, ce qui augmente d'autant la durée de réalisation des projets et peut même influencer leur rentabilité, voire en bloquer la réalisation. Par exemple, entre la parution du décret du second appel d'offres en 2005 et l'annonce d'Hydro-Québec des soumissionnaires retenus en mai 2008 au Québec, il s'est passé 2 ans et demi. Ces longueurs procédurales participent à retarder le développement de l'énergie éolienne au Québec et expliquent en partie pourquoi certains projets ont dû mettre la clé sur la porte. Entre-temps, les conditions de crédits se sont dépréciées et le prix des machines a augmenté pour les développeurs, ce qui se traduit par des coûts d'investissements plus élevés pour le projet et un prix obtenu lors de l'appel d'offres qui lui n'augmente pas.

\section{Critères 2 et 4: Innovations technologiques, industries domestiques, bénéfices économiques et diversité technologique}

Il peut être plus efficace pour réduire les coûts des technologies de les introduire rapidement et en fort volume par un tarif suffisamment intéressant qui les amène rapidement sous leur courbe d'apprentissage (cas des tarifs d'achat garanti) plutôt que vouloir introduire lentement les technologies et réduire lentement leurs coûts.

\section{Les tarifs d'achat garanti sont efficaces pour stimuler une technologie jusqu'à ce qu'il y ait un marché compétitif. Ils soutiennent une vitesse de diffusion élevée qui offre aux manufacturiers la certitude d'un marché local et durable pouvant conduire à la réussite de la filière industrielle.}

Les tarifs d'achat garanti sont efficaces pour stimuler une technologie jusqu'à ce qu'il y ait un marché compétitif. Ils soutiennent une vitesse de diffusion élevée qui offre aux manufacturiers la certitude d'un marché local et durable pouvant conduire à la réussite de la filière industrielle ${ }^{\mathrm{a}}$. Avec les tarifs d'achat garanti, les profits sont redistribués aux producteurs. Ils l'investissent en Recherche et Développement privée, ce qui réduit les coûts, améliore leurs profits et favorise l'innovation. Cette situation explique pourquoi 7 des 10 premiers turbiniers étaient originaires de pays qui avaient bénéficié de tarifs d'achat garanti en $2004^{8}$. En 2009, cette proportion est toujours de 8 sur 10 , mais les noms ont changé : des manufacturiers chinois ont intégré ce peloton de tête, avec là encore un tarif d'achat garanti ou assimilé depuis 2005. Enfin, soulignons que les tarifs d'achat garanti favorisent une diversité technologique, car les tarifs peuvent être facilement différenciés en fonction des technologies ${ }^{6}$.

Avec les QUOTAS, les surplus vont au consommateur et le producteur ne reçoit pas forcément assez de profits pour investir en R\&D et réduire ses coûts. Dans le même temps, la pression du coût minimum encourage les producteurs à se tourner vers des technologies étrangères et existantes. Comme l'évolution des politiques par QUOTAS s'effectue souvent en dents de scie, on n'a pas un développement continu qui encouragerait en retour des innovations. Cela rend difficile l'établissement d'une industrie nationale parce que les investissements se réalisent dans une perspective à court terme, ce qui limite aussi la création d'emplois et des bénéfices économiques associés ${ }^{6}$. 
Au Québec, les appels d'offres ont permis de créer un volume intéressant pour permettre l'établissement de manufacturiers extérieurs au pays, mais seulement pour certaines composantes de l'éolienne (la tour, la nacelle ou les pales). On ne fabrique pas les contenus à haute valeur ajoutée. Cela peut s'expliquer par un problème de positionnement tardif sur le marché éolien. L'éolien a commencé dans les années 2000 alors que cela fait près de vingt ans que la technologie est utilisée à large échelle au Danemark et en Allemagne. On a donc eu le temps d'améliorer et de développer les technologies. Ce sont d'ailleurs des technologies allemandes qui ont été utilisées dans le cadre du second et du troisième appel d'offres. À contrario, le seul manufacturier québécois avec une licence allemande, AAER, n'ayant que très d'expérience, n'a obtenu aucun contrat dans le cadre des différents appels d'offres : il a donc fini par faire faillite.

À plus long terme, le développement par «à coups » des appels d'offres au Québec créé un climat d'incertitude pour l'industrie qui réclamait, lors du colloque CANWEA 2010, une certaine visibilité et stabilité des quotas éoliens au Québec pour l'après 2015. Faute d'une stabilité à moyen et long terme du marché intérieur québécois, les industriels manifestaient leur intention d'aller exporter leur savoir-faire ailleurs et notamment en Ontario, où le marché éolien suscité par la politique tarifaire actuelle du «green energy act» vise l'implantation de 700 à $1000 \mathrm{MW} \mathrm{/} \mathrm{an}$ d'éolien. Cette dynamique tournée vers les énergies renouvelables se traduit aussi en termes d'emplois « verts ». En 2 ans, l'Ontario a déjà créé 13000 emplois et est aussi devenu le leader pancanadien en matière d'innovation dans les technologies vertes. D'ici fin 2012, elle prévoit près de 50000 emplois. On est loin des 1600 emplois directs (ou 5000 emplois indirects induits) créés par la filière éolienne en Gaspésie.

\section{Critère 3 : L'acceptabilité sociale}

Les appels d'offres compliquent l'acceptabilité sociale des projets. Au contraire, l'acceptabilité sociale des projets éoliens est beaucoup plus facile avec des tarifs d'achat garanti, et ceci pour plusieurs raisons. D'abord, les appels d'offres ont une influence directe sur la qualité et le contenu du processus décisionnel. Dans le contexte compétitif d'un appel d'offres, il est difficile pour un promoteur de consulter et d'informer la population de manière exhaustive ou transparente avant que le projet ne soit accepté par Hydro-Québec. C'est pourquoi les consultations commencent souvent lorsque le projet est accepté, alors que les principales caractéristiques des projets sont déjà décidées, telles que l'envergure du projet ou la localisation des éoliennes. D'autre part, la contrainte de temps imparti fait en sorte de rajouter une pression supplémentaire pour le milieu local, lequel doit prendre des décisions ou faire des ententes négociées avec un promoteur dans un délai imparti qui ne lui convient pas forcément.

\section{En Allemagne, au début du développement éolien dans les années 90 (AO à l'époque), les projets s'implantaient le long de la côte de la mer du Nord, la zone la plus ventée. Depuis que les tarifs d'achat garanti ont été implantés, les projets se sont dispersés sur l'ensemble du territoire y compris à l'intérieur des terres. À l'échelle même de l'Europe, il est étonnant de voir que ce pays, possédant l'un des moins bons gisements éoliens, a longtemps été l'un des premiers pays en termes de capacité éolienne installée.}

Ainsi, dans un appel d'offres, les projets éoliens sont concentrés dans les zones les plus économiquement rentables. Au Québec, cela signifie un déploiement en région habitée souvent proche des zones valorisées par les populations locales. Au contraire, les tarifs d'achat garanti sont différentiables géographiquement (avec des tarifs avancés en énergie renouvelable), ce qui se traduit par une plus grande dispersion des projets sur le territoire. Si on se rend compte que la rentabilité du parc est plus importante que prévue, les tarifs décroissent, ce qui évite aux développeurs de faire des profits indus. Si au contraire le site s'avère moins bon que prévu, les tarifs restent au même niveau. En Allemagne, au début du développement éolien dans les années 90 (AO à l'époque), les projets s'implantaient le long de la côte de la mer du Nord, la zone la plus ventée. Depuis que les tarifs d'achat garanti ont été implantés, les projets se sont dispersés sur l'ensemble du territoire y compris à l'intérieur des terres. À l'échelle même de l'Europe, il est étonnant de voir 
que ce pays, possédant l'un des moins bons gisements éoliens, a longtemps été l'un des premiers pays en termes de capacité éolienne installée ${ }^{6}$.

Au Québec, le premier appel d'offres était dédié à la région de la Gaspésie. À cause du moindre coût, les projets sont situés le long du pourtour gaspésien, dans les meilleurs gisements éoliens, proches des lignes électriques, en parfaite adéquation avec les corridors touristiques ou migratoires. Dans la MRC de Matane, la situation est préoccupante, car quatre parcs éoliens devraient coexister sur une superficie de $20 \mathrm{~km}^{2}$. Plusieurs craignent l'impact cumulatif de ces projets. Lorsqu'on a ouvert le territoire à l'ensemble du Québec dans le second appel d'offres, les projets se sont là encore installés dans les régions proches des lieux de consommation d'énergie pour réduire les coûts de raccordement. En Chaudière-Appalaches ou en Montérégie par exemple, cela coïncide avec des zones plus densément peuplées où de nombreux néo-ruraux se sont installés pour profiter de la campagne. Cela peut créer des conflits d'usage et représente une source d'opposition sociale dans plusieurs localités. On est bien loin du territoire du Grand Nord québécois où il n'y a personne, mais où les coûts de raccordement sont élevés.

Ensuite, les appels d'offres favorisent des parcs privés de grandes tailles avec peu de retombées locales. Avec des tarifs d'achat garanti, les projets sont de tailles diversifiées, favorisent une certaine justice sociale dans le choix des promoteurs et favorisent la distribution de retombées locales acceptables. Dans les appels d'offres, la nécessité du moindre coût encourage la réalisation de grands projets pour des raisons d'économies d'échelle; cet élément complique évidemment l'insertion paysagère des projets. Par exemple, la taille moyenne des projets pour les deux premiers appels d'offres au Québec était de 70 machines. Au contraire, pour les tarifs d'achat garanti, comme le prix est fixé d'avance et que l'accès au réseau est possible pour n'importe quel type d'entreprise ou de promoteur, il existe une certaine équité du mode de propriété. Les petites compagnies sont favorisées au même titre que les grandes entreprises, ce qui conduit à une variété de tailles de projets et de compagnies. En France par exemple, la plupart des projets sont compris entre 6 et $30 \mathrm{MW}$; un parc de $50 \mathrm{MW}$ est considéré comme un projet d'envergure. On observe aussi diverses tailles d'entreprises privées françaises ou étrangères sur le territoire.

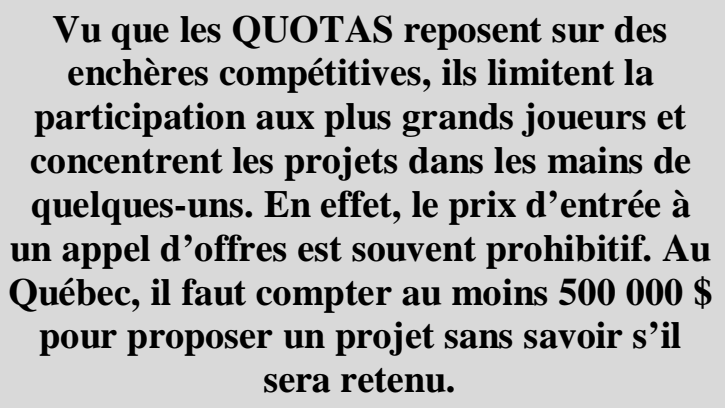

De plus, vu que les QUOTAS reposent sur des enchères compétitives, ils limitent la participation aux plus grands joueurs et concentrent les projets dans les mains de quelques-uns. En effet, le prix d'entrée à un appel d'offres est souvent prohibitif. Au Québec, il faut compter au moins 500000 \$ pour proposer un projet sans savoir s'il sera retenu. De plus, soumettre un projet ne suffit en général pas. Pour avoir une chance de l'emporter, il faut proposer un portefeuille de projets pour être capable de mitiger le risque. Dans ce contexte, les petits projets (et les petits promoteurs) sont incapables de compétitionner avec de plus grands promoteurs. Au Royaume-Uni, $70 \%$ des installations éoliennes sont détenues par seulement quatre distributeurs d'électricité. Au Québec, la plupart des 24 projets acceptés par Hydro-Québec dans le cadre des deux premiers appels d'offres sont détenus majoritairement par des consortiums ou multinationales extérieurs au pays. Dans l'appel d'offres communautaire, une seule coopérative a été acceptée et un seul projet $100 \%$ municipal a pu se former. La plupart des autres projets sont des partenariats public-privé majoritairement privé.

Au Royaume-Uni, 70 \% des installations éoliennes sont détenues par seulement quatre distributeurs d'électricité. Au Québec, la plupart des 24 projets acceptés par Hydro-Québec dans le cadre des deux premiers appels d'offres sont détenus majoritairement par des consortiums ou multinationales extérieurs au pays. 
Enfin, avec des QUOTAS, la pression du coût minimum ne permet pas aux développeurs de distribuer beaucoup d'argent aux collectivités locales. C'est d'ailleurs une des raisons qui expliquait le mécontentement observé au Québec en 2006-2007. Certaines personnes jugeaient les retombées locales et régionales insatisfaisantes. C'était le cas aussi bien des redevances ou contributions volontaires versées aux municipalités que des loyers perçus par les agriculteurs. À cette époque, ces retombées ne représentaient chacune que quelques milliers de dollars par MW. Au-delà de cette question des redevances, beaucoup de personnes considéraient aussi que la plupart des profits étaient distribués aux actionnaires alors que la population locale ne présentait que des «impacts». Ainsi, ces retombées économiques réelles (profits compris) ne représenteraient finalement que $1 \%$ des coûts globaux du projet.

\section{Critère 5 : Coûts, les prix et la concurrence}

\section{Les QUOTAS auraient plutôt tendance à faire augmenter les tarifs, car les meilleurs sites sont exploités en premier et les sites les moins rentables en dernier. C'est ce que l'on a observé au Québec : le prix obtenu pour les appels d'offres est de plus en plus cher au fil des années $(6.5 \$ \$ / \mathrm{kWh}$ en 2005 , $8.7 \phi \$ / k W h$ en 2009 , et $11.3 \phi \$ / k W h$ en 2010).}

Les coûts dépendent de la manière dont la politique tarifaire est structurée et du détail de ces systèmes tarifaires. En moyenne, les pays avec des tarifs d'achat garanti ont des prix plus bas que ceux avec les quotas, et ce, en dépit du fait qu'ils présentent parfois moins de ressources $^{\mathrm{b}}$ pour diverses raisons. D'abord, comme les tarifs d'achat garanti renforcent le développement des industries nationales de fabrication, ils encouragent l'établissement de nouvelles entreprises. En soutenant l'industrie, ils favorisent la création d'emplois et la croissance économique. De nombreuses entreprises pénètrent le marché et créent une compétition sur les prix de vente de l'énergie éolienne. Ainsi, depuis 1991, les prix de vente par kWh ont baissé de $55 \%$ en Allemagne.
Ensuite, la diffusion rapide des tarifs d'achat garanti se traduit par des économies de volume qui se répercutent sur une réduction du prix des turbines et des prix de vente de l'énergie éolienne d'année en année. Par exemple, le prix des turbines a diminué de $50 \%$ entre 1994 et 2004 en Allemagne. Au contraire, les QUOTAS auraient plutôt tendance à faire augmenter les tarifs, car les meilleurs sites sont exploités en premier et les sites les moins rentables en dernier. C'est ce que l'on a observé au Québec : le prix obtenu pour les appels d'offres est de plus en plus cher au fil des années $(6.5 \phi \$ / \mathrm{kWh}$ en $2005,8.7 \phi \$ / \mathrm{kWh}$ en 2009 , et $11.3 \phi \$ / \mathrm{kWh}$ en 2010) ${ }^{c}$. Enfin, les tarifs d'achat garanti ne sont pas forcément plus coûteux (par $\mathrm{kWh}$ généré) que les QUOTAS. Le gouvernement allemand estime le coût supplémentaire moyen à $12 € / a n$ et par chaque ménage. Cela s'explique en partie par la facilité d'administration des tarifs d'achat garanti, qui ne demande que de fixer et d'ajuster régulièrement les tarifs.

\section{Critère 6: Sécurité financière et risque de financement}

Les plus hauts risques et les plus bas profits associés aux QUOTAS rendent ces systèmes moins attractifs pour les investisseurs que les tarifs d'achat garanti. Avec les tarifs d'achat garanti, les prix de vente de l'énergie sont connus d'avance sur 15 ou 20 ans. Cela rassure les compagnies qui désirent investir et les incite à agir dans une perspective à long terme. Avec cette certitude, il est aussi plus facile d'obtenir du financement de la part des banques, car elles ont la garantie d'un retour d'investissement sur une période de temps donnée. L'évaluation du risque et le financement des coûts d'avant-projet est en quelque sorte facilité car on connait quasiment tous les paramètres du projet. Avec les QUOTAS, les promoteurs doivent évaluer les tarifs auxquels ils vont soumissionner leur projet. Cette évaluation est confrontée à de nombreuses incertitudes potentielles, tout en n'étant pas sûr que son projet soit au final accepté : c'est donc dans bien des cas de l'argent dépensé en pure perte ${ }^{6}$.

La plus grosse incertitude est certainement sur les prix moyens de l'électricité qui vont sortir de 
l'appel d'offres. Souvent, les appels d'offres incitent les soumissionnaires à proposer le plus bas coût possible pour être retenus, ce qui fait qu'au moment de réaliser son projet on ne peut plus le faire, car il a été soumis trop bas. C'est ce qui est arrivé en France dans les années 2000 lors du programme Éole 2005 où seulement quelques MW ont été construits pour un appel à proposition de $250 \mathrm{MW}$. L'incertitude sur l'avenir de l'offre et la demande pendant la durée de vie du projet est aussi importante. En effet, la demande est créée par des objectifs politiques qui peuvent changer; estimer l'offre est aussi délicat, car c'est un processus complexe qui dépend de beaucoup de facteurs comme le prix des matières premières, le retard sur les projets, etc. Les coûts de préparation des soumissions d'appel d'offres sont élevés ce qui ajoute un élément de risque et d'incertitude que peu de promoteurs peuvent se permettre. Enfin, l'incertitude des subventions gouvernementales peut conduire à des risques plus élevés pour les investisseurs, nécessitant un plus haut taux de retour. Au Canada, c'est un peu ce que l'on a

\section{CONCLUSION}

\section{Les tarifs d'achat garanti s'avèrent être les meilleurs outils tarifaires pour développer les énergies renouvelables, notamment pour les petits projets communautaires en région habitée. C'est le souhait de la plupart des acteurs issus des milieux communautaire et coopératif, mais c'est aussi le souhait de plusieurs acteurs de l'industrie.}

La plus grande difficulté de l'implantation d'un tarif d'achat garanti réside dans le choix des tarifs. Trop haut, il peut provoquer un emballement de l'offre et induire un déficit public important. Trop bas, cela devient des incitatifs insuffisants pour établir durablement les filières considérées. Il y a donc un équilibre à trouver pour un prix «juste». Évidemment, cet équilibre dépend des coûts de production réels des filières énergétiques et du contexte énergétique national: il ne sera pas le même pour l'éolien que pour le photovoltaïque, ou entre l'Ontario et le Québec. En effet, l'Ontario vit une pénurie énergétique majeure avec des observé en 2010-2011 avec le nonrenouvellement du programme Écoénergie accordant $1 \phi / \mathrm{kWh}$ aux promoteurs éoliens.

\section{Critère 8 : Flexibilité}

Avec les tarifs d'achat garanti, il est possible d'ajuster les tarifs suivant une base régulière pour refléter les changements technologiques et les conditions de marché. Par contre, une fois que les QUOTAS et les calendriers sont établis, il devient difficile de les ajuster. Même si les conditions du marché changent ou que les technologies évoluent, connaissant des percées importantes en efficacité et/ou de coût, il est difficile que le calendrier soit modifié ou que les objectifs deviennent plus ambitieux sans des délais supplémentaires. Avec des tarifs d'achat garanti, les objectifs de réalisation peuvent être dépassés. Dans les QUOTAS, même si l'on connaît d'avance la quantité que l'on va implanter, il n'est pas possible de dépasser les objectifs s'ils ne sont pas assez ambitieux ${ }^{6}$.

coûts élevés de l'électricité. Au contraire, le Québec présente des prix de l'électricité beaucoup plus faibles et devrait vivre d'ici 2025 des surplus électriques majeurs. Ses besoins additionnels en électricité sont donc moins importants qu'en Ontario, cela devrait donc se répercuter sur des tarifs moins élevés.

Malgré cette difficulté, les tarifs d'achat garanti s'avèrent être les meilleurs outils tarifaires pour développer les énergies renouvelables, notamment pour les petits projets communautaires en région habitée. C'est le souhait de la plupart des acteurs issus des milieux communautaire et coopératif, mais c'est aussi le souhait de plusieurs acteurs de l'industrie. Dans un document d'orientation de 2008, l'Association canadienne de l'énergie éolienne (CANWEA), définit clairement les tarifs de rachat comme «le meilleur système tarifaire à privilégier pour les énergies renouvelables, dont l'Amérique du Nord pourrait s'inspirer, car ils sont tirés des meilleures pratiques de par le monde ». L'Ontario a ouvert la voie et a joué le rôle de pionnier en instaurant des tarifs d'achat intéressants pour les différentes 
énergies renouvelables depuis mai 2009. Depuis, treize États américains et trois provinces canadiennes ont suivi le pas ou sont en train de le faire. Le Royaume-Uni, l'un des plus fervents défenseurs des quotas en Europe, a lui aussi décidé en 2010 de passer à une tarification fixe pour des projets inférieurs à $5 \mathrm{MW}$. La Chine, elle aussi, est devenue en 2010 le premier marché éolien mondial, en particulier grâce à des tarifs d'achat garanti.

Bref, les exemples ne manquent pas et montrent l'engouement que suscite le modèle des tarifs d'achat partout dans le monde. Alors, pourquoi rester dans une logique du tout-appel d'offres au Québec? À la lumière de ce que l'on vient de dire, l'avenir énergétique québécois semble plutôt se tourner vers une solution tarifaire « mixte», à savoir des projets d'énergies renouvelables communautaires et de petites tailles en région habitée et des grands projets possiblement par appel d'offres dans le Grand Nord du Québec. Si le Québec veut rester à l'avant-garde dans le domaine des énergies renouvelables, il devrait suivre le vent qui souffle en Amérique du Nord, dans beaucoup de pays européens et partout ailleurs dans le monde surtout dans un contexte où l'hydroélectricité devient du même ordre de rentabilité que l'éolien. Si cela ne se fait pas, ce ne sera pas par manque de potentiel énergétique ou absence de vision des acteurs locaux, ce sera plutôt par manque de vision politique à long terme.

\section{Si le Québec veut rester à l'avant-garde dans le domaine des énergies \\ renouvelables, il devrait suivre le vent qui souffle en Amérique du Nord, dans beaucoup de pays européens et partout ailleurs dans le monde surtout dans un contexte où l'hydroélectricité devient du même ordre de rentabilité que l'éolien.}

Un changement culturel important est requis. Depuis les fondements d'Hydro-Québec, la vision énergétique du développement énergétique est orientée uniquement vers le consommateur car on souhaite développer l'énergie au meilleur coût pour l'ensemble de la société. Cet objectif est louable, mais il y aurait certainement lieu de repositionner le débat à l'ère du $\mathrm{XXI}^{\mathrm{e}}$ siècle, de la libéralisation de l'énergie d'un côté et de la volonté de réaliser un «développement durable » des énergies et des territoires de l'autre. Depuis les années 2000, les Québécois reçoivent des dividendes pour les revenus excédentaires d'Hydro-Québec. Dans le même temps, on fait construire de gros parcs éoliens par des multinationales privées et on ne permet que très peu aux collectivités de tirer des bénéfices de ce développement. N'y aurait-il pas lieu d'équilibrer un peu cette logique davantage vers le «producteur et les collectivités locales »? Est-il logique, que des collectivités locales qui se dévitalisent soient dépossédées de leurs ressources et ne puissent que difficilement participer au développement du vent?

En retrouvant un certain équilibre, on pourrait se servir davantage de l'éolien comme levier de développement, pour et par les collectivités locales qui habitent le territoire. Évidemment, le troisième appel d'offres communautaire a ouvert la voie à une nouvelle manière de faire du développement en limitant la taille des parcs à $25 \mathrm{MW}$ en région habitée et en rendant obligatoire un contrôle local minimum de $30 \%$. Cependant, de nombreuses questions subsistent et un modèle tarifaire par tarif d'achat garanti serait préférable pour de nombreuses raisons, ne serait-ce que pour offrir la possibilité d'avoir des partenariats avec les entrepreneurs privés (lorsque le capital local investi est inférieur à $50 \%$, on ne contrôle pas véritablement ce qui se passe sur le terrain), de limiter la compétition entre les collectivités locales dans des appels d'offres communautaires et d'éviter des gaspillages de temps et d'argent pour beaucoup de projets qui ne se feront pas!

On fait construire de gros parcs éoliens par des multinationales privées et on ne permet que très peu aux collectivités de tirer des bénéfices de ce développement. N'y auraitil pas lieu d'équilibrer un peu cette logique davantage vers le « producteur et les collectivités locales »? Est-il logique, que des collectivités locales qui se dévitalisent soient dépossédées de leurs ressources et ne puissent que difficilement participer au développement du vent? 


\section{BIBLIOGRAPHIE}

${ }^{1}$ Ministère des Ressources naturelles de la Faune (MRNF) (2006) L'énergie pour construire le Québec de demain, la stratégie énergétique du Québec : 2006-2015. Québec, MRNF.

${ }^{2}$ Francoeur, L.-G. (2011) Le Devoir - Énergies vertes : Hydro doit offrir des prix garantis-Le rapport de l'équipe spéciale propose une nouvelle stratégie de développement. Montréal,

${ }^{3}$ Brassard, M.-J., et al. (2012) Le Devoir - Le développement des énergies renouvelables a un coût : pourquoi ne pas le déterminer et le garantir ? Montréal, Le Devoir.

${ }^{4}$ Gipe, P. (2005) Renewable Energy Policy Mechanisms. Tehachapi.

${ }^{5}$ Hvelplund, F. (2001) Political Prices or Political Quantities? - A Comparison of Renewable Energy Support Systems. New energy, 2001 (5): 18-23.

${ }^{6}$ Sawin, J. L. (2004). National Policy Instruments Policy Lessons for the Advancement \& Diffusion of Renewable Energy Technologies Around the World. Dans Secrétariat of the Internationl Conference for Renewable Energies (Éd.), International Conference for Renewable Energy, p. 56. Bonn, janvier 2004.

${ }^{7}$ Fouquet, D. et al. (2005) Reflections on a Possible Unified EU Financial Support Scheme for Renewable Energy Systems : a Comparison of Minimum-price and Quota Systems and an Analysis of Market Conditions. Brussels et Washington DC, European Renewable Energies Federation and Worldwatch Institute. Saucier, C. et al. (2009) Rapport final de l'Unité de recherche sur le développement territorial durable et la filière éolienne. Des installations éoliennes socialement acceptables : élaboration d'un modèle d'évaluation de projets éoliens dans une perspective de développement territorial durable. Rimouski, Université du Québec à Rimouski.

${ }^{8}$ Lewis, J. I. et Wiser, R. H. (2007) Fostering a Renewable Energy Technology Industry: An International Comparison of Wind Industry Policy Support Mechanisms. Energy Policy, 35 (3): 18441857.

\section{Notes}

${ }^{a} A i n s i$, le positionnement industriel est fortement influencé par le moment d'entrée sur le marché et l'existence d'un marché intérieur suffisant et stable.

${ }^{b}$ La comparaison de l'Allemagne et du Royaume-Uni est frappante. En 2004, les prix étaient entre 6.6 et $8.8 \notin \$ / \mathrm{kWh}$ en Allemagne avec le potentiel éolien le moins élevé d'Europe. Ils sont de 9.6 et $8.8 \phi \$ / \mathrm{kWh}$ en moyenne au Royaume-Uni, alors que son potentiel éolien est le plus élevé d'Europe.

'Prix donnés avant transport et équilibrage. 